\title{
A RANDOMIZED CONTROLLED STUDY OF INGUINAL HERNIA USING MONOFILAMENT NON-ABSORBABLE SUTURE VERSUS MONOFILAMENT ABSORBABLE SUTURE IN LICHTENSTEIN TENSION FREE HERNIA REPAIR
}

\author{
Dr Hamikchandra \\ Patel $^{*}$ \\ Dr Karan Patel \\ Senior Resident,Dept Of Surgery ,Govt Medical College, Vadodara. \\ ${ }^{*}$ Corresponding Author \\ Senior Resident,Dept Of Surgery ,Govt Medical College, Vadodara.
}

ABSTRACT Background: The surgical treatment of inguinal hernias has evolved through several stage, history of groin hernia is the history of surgery itself. But, chronic pain is still a major irritating post-operative complain of the patient. The objective of the present study was to compare post-operative outcomes of mesh fixation with monofilament non-absorbable v/s monofilament absorbable suture material in terms of postoperative pain, chronic groin pain, seroma formation, wound infection and recurrence in Lichtenstein hernioplasty. Methods: This is a single centre, prospective randomized controlled study of 152 cases of inguinal hernia comparing post-operative outcomes of mesh fixation with monofilament non-absorbable v/s monofilament absorbable suture material in Lichtenstein hernioplasty at Baroda Medical College and S.S.G. Hospital, between lst December 2017 to 30th November 2018. Results: Total 152 patients of unilateral or bilateral inguinal hernia were studied 76 in each group. Chronic groin pain mean visual analogue scale score at 3 months was higher in group with non-absorbable suture compared to monofilament absorbable group $(1.3 \pm 0.9 \mathrm{v} / \mathrm{s} 0.95 \pm 0.8 \mathrm{pvalue}<0.05)$. Conclusions: Monofilament absorbable suture is associated with less chronic groin pain and compared to monofilament nonabsorbable sutures.

\section{KEYWORDS : Inguinal hernia, Lichtenstein's hermioplasty, Absorbable suture, Non-absorbable suture, Chronic} groin pain

\section{INTRODUCTION}

An inguinal hernia is defined as a protrusion of a viscous or a part of a viscous into the inguinal canal either through deep ring or through Hesselbach's triangle. The surgical treatment of inguinal hernias has evolved through several stages history of groin hernia is the history of surgery itself. ${ }^{1}$ Since the time Bassini described his technique an ideal hernia repair should be tension free, tissue based, with no potential damage to vital structures, no long term pain or complications and no recurrence. Inguinal hernia repair is one of the most common operations performed in general surgery. Chronic inguinal pain occurs in $16-60 \%$ patients post-operatively. Irrespective of its mild intensity, it substantially affects quality of life of the patient. $^{2,3}$

\section{OBJECTIVE}

The objective of the present study was to compare postoperative outcomes of mesh fixation with monofilament nonabsorbable suture material $\mathrm{v} / \mathrm{s}$ monofilament absorbable suture material in Lichtenstein tension free hernioplasty in terms of postoperative pain assessment using visual analogue scale (VAS) score; chronic groin pain; seroma formation; wound infection; recurrence rate.

\section{METHODS}

This is a single center, prospective randomized controlled study of 152 cases of inguinal hernia comparing postoperative outcomes of mesh fixation with monofilament nonabsorbable v/s monofilament absorbable suture material at Baroda Medical College and S.S.G. Hospital, between lst December 2017 to 30th November 2018, all the cases of uncomplicated inguinal hernia with age above 18 years were included. After explaining the purpose of the study and the methods of the treatment in their own language, written informed consent to participate in the study was taken after taking detailed history including age, chief complaints and duration, other associated conditions like chronic cough, chronic constipation, urinary complaints etc, history of previous abdominal surgeries, family history, occupation, marital status etc. detailed physical examination was conducted and diagnosis of primary inguinal hernia was made and all were admitted in surgical ward after all basic investigations Lichtenstein tension free repair was planned after using blind envelope method for randomization. In
Group A, mesh fixation was done by non-absorbable suture material polypropylene $(n=76)$ and Group $B$, mesh fixation was done by monofilament absorbable suture material poliglecaprone $(n=76)$. Pre-operative antibiotic (Inj. amoxycillin+clavulanic acid $1.2 \mathrm{gms}$ intravenously) was given 30 minutes before putting skin incision. Telephonic contact numbers and details address were collected for follow up.

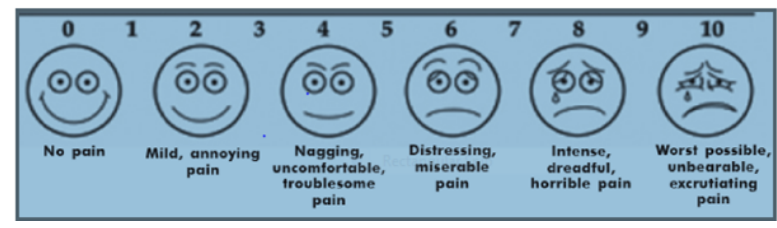

Figure 1: VAS used for assessment of pain.

0: No pain; 1, 2, 3: Mild pain; 4, 5, 6: Moderate pain; 7, 8, 9: Severe pain; 10: Worst imaginable pain.

Follow up visits were at tenth day, at one month, and at third month after surgery either on outpatient department basis or by telephonic conversations. Total post-operative follow-up period was 3 month. Same post-operative protocol for treatment is followed in all the cases which includes and postoperative two doses of (Inj. amoxycillin+clavulanic acid 1.2 gms intravenously) along with analgesic (inj. diclofenac 50 mg intravenously 12 hourly) and Inj. pantoprazole $40 \mathrm{mg}$ intravenously 12 hourly in all the cases. Patients were asked to ambulate as early as possible after effect of spinal anesthesia weans off. Patients were started orally after 6 hours of operation. From first post-operative day, after starting orally all the patient were switched over to oral capsule amoxicillin+clavulanic acid $625 \mathrm{mg}$ three times a day along with tablet diclofenac $50 \mathrm{mg}$ twice a day and tablet pantoprazole $40 \mathrm{mg}$ once a day till 5 th post-operative day.

From sixth post-operative day, antibiotic and analgesics are continued if pain persist or any sign of infection was observed. All the patients were observed for post-operative pain (VAS), wound hematoma, seroma formation, wound infection. All the cases underwent routine dressing on 2nd post-operative day, 5th post-operative day and 10th post-operative day with suture removal. During dressing, any swelling, discharge, discoloration at wound site if present was documented. If 
done. Hematoma or seroma was considered in patients having anechoic collection with or without internal echoes respectively in ultrasonographic findings. Epididymo-orchitis was considered in patients having bulky heterogenous echotexture with increased internal vascularity. Postoperatively, early recurrence and chronic pain if present was also documented. In our study, Patients were assessed for post-operative pain using VAS score on daily basis till 5 th post-operative day then at 10th day, 1 and 3 months after surgery. Patient has to point on the scale the amount of pain he is currently experiencing. In contrast, chronic pain is defined as pain persisting beyond 3 months.4-6 Results were analyzed using MedCalc Software version 12.5.0 and Microsoft Excel was used to generate graphs and tables. Student t test and Chi-square or Fisher exact test has been used to find the significance of study parameters on categorical scale between two or more groups with suggestive significance $(+)$ means $\mathrm{p}$ value as $0.05<\mathrm{p}<0.10$. Moderately significant with $\mathrm{p}$ value as $0.01<\mathrm{p}<0.05$ and strongly significant means $p$ value as $\mathrm{p}<0.01$ respectively.

\section{RESULTS}

Out of 152 patients 74 were having right inguinal hernia, 49 patients with left inguinal hernia and 29 patients were having bilateral inguinal hernia. We analyzed post-operative pain using VAS score on daily basis till 5 th post-operative day and then at 10th post-operative day. On post-operative day 1 the mean VAS score in group A was 5.56 \pm 1.09 , while that in group $B$ was $4.88 \pm 0.97$, though the difference is small it is still statistically significant with a $p$ value of 0.02 . On postoperative day 3 the mean VAS score in group A was 4.12 \pm 0.95 , while that in group B was $3.48 \pm 0.77$, which was statistically significant with a p value of $<0.04$. On post-operative day 5 the mean VAS in Group A was 2.60 0.70 , while that in Group B was $1.92 \pm 0.81$. This difference is not statistically significant with $a p$ value of 0.21 . And on day 10, the mean VAS score in group A was $2.5 \pm 0.59$, while that in group $B$ was $1.5 \pm 0.22$, with a p value of 0.046 which is statistically significant. Overall, Group B experienced less pain compared to group A. Postoperative complication we observed seroma in 6 out of $76(8 \%)$ patients in group $A$ and 9 out of 76 (12\%) patients in group B. For this, $\mathrm{p}$ value is 0.69 , which is statistically not significant. Scrotal swelling developed in 12 out of 76 (16\%) patients in group A and 9 out of 76 (12\%) in up B. For this, p value is 0.59 , which is considered statistically not significant.

Table 1-Post operative pain (VAS Scale)

\begin{tabular}{|l|l|l|l|}
\hline Days & Group A & Group B & P value \\
\hline 1 & $5.56 \pm 1.09$ & $4.88 \pm 0.97$ & 0.021 \\
\hline 2 & $4.60 \pm 1.08$ & $4.24 \pm 0.87$ & 0.20 \\
\hline 3 & $4.12 \pm 0.95$ & $3.48 \pm 1.00$ & 0.04 \\
\hline 4 & $3.44 \pm 0.91$ & $2.33 \pm 0.76$ & 0.114 \\
\hline 5 & $2.6 \pm 0.70$ & $1.92 \pm 0.81$ & 0.21 \\
\hline 10 & $2.5 \pm 0.59$ & $1.5 \pm 0.22$ & 0.046 \\
\hline
\end{tabular}

Table 2-Early Complications in two groups of patients

\begin{tabular}{|c|c|c|c|c|c|c|c|}
\hline \multicolumn{2}{|l|}{ Complications } & \multicolumn{2}{|c|}{$\begin{array}{c}\text { Group A } \\
(\mathrm{n}=76)\end{array}$} & $\begin{array}{c}\text { Group B } \\
(\mathrm{n}=76)\end{array}$ & \multicolumn{2}{|c|}{$\begin{array}{c}\text { Total } \\
(n=152)\end{array}$} & \multirow[t]{2}{*}{ P value } \\
\hline $\mathbf{N}$ & $\%$ & & & $\mathbf{N}$ & $\%$ & & \\
\hline Seroma & 6 & 8 & 9 & 12 & 15 & 9 & 0.69 \\
\hline Wound Infection & 3 & 4 & 6 & 8 & 9 & 6 & 0.67 \\
\hline Local swelling & 0 & - & 0 & - & 0 & - & - \\
\hline
\end{tabular}

Table 3-Late complication-Chronic Pain

\begin{tabular}{|l|l|l|}
\hline Method & At 1 Month & Ät 3 Month \\
\hline Group A (n=76) & $15(20 \%)$ & $10(12 \%)$ \\
\hline Group B (n=76) & $8(10 \%)$ & $3(4 \%)$ \\
\hline VAS Group A & $1.7 \pm 0.4$ & $1.30 \pm 0.9$ \\
\hline VAS Group B & $1.1 \pm 0.5$ & $0.95 \pm 0.8$ \\
\hline P value & 0.049 & 0.048 \\
\hline
\end{tabular}

Table 4-Incidence of Recurrence

\begin{tabular}{|l|l|}
\hline Method & Recurrence \\
\hline Group A & 0 \\
\hline Group B & 0 \\
\hline
\end{tabular}

Wound infection was observed in 3 out of 76 (4\%) patients in group A and 6 out of 76 (8\%) in the group B. The p value is 0.67 , which is not statistically significant. All the patients of both groups were followed after discharge for a period of 3 months with regular outpatient department checkups at 10th day, 1 month and 3 months. At 1 month, 15 (20\%) patients of group A $(n=76)$ complained of disturbing groin pain at site of surgery during routine activities, while $8(10 \%)$ patient in group B was having similar complaint. Pain was managed by oral analgesic tablet diclofenac $50 \mathrm{mg}$ twice a day. At 3 months after surgery, $10(12 \%)$ patients of group $A(n=76)$ and $3(4 \%)$ patient of group $B(n=76)$ patients complained of groin pain which persisted despite of oral analgesics. Mean VAS score at 1 month in group A was $1.7 \pm 0.4$ and in group B was $1.1 \pm 0.5$ and at $3 r d$ month it was $1.3 \pm 0.9$ in group $A$ and $0.95 \pm 0.8$ in group $B$. The $p$ value for pain at one and three month postoperative periods between the two groups are statistically significant $(p<0.05)$. No recurrence in either of the groups during this study period. However, long term follow-up is required to judge the late recurrence rate.

\section{DISCUSSION}

Use of prosthetic mesh for inguinal hernia repair has become common practice decreasing the rate of recurrence. ${ }^{7.8}$ Morbidity associated with a tension-free mesh repair consists mainly of chronic groin pain that occurs in $16 \%$ to $62 \%$ of patients. ${ }^{1}$ Factors being irritation of inguinal nerves by sutures or mesh, or an inflammatory reaction to the mesh, or simple tissue scaring. ${ }^{9,10}$ The results were compared to various other studies done in this field. Igor et al found that mean age in group A was $47 \pm 19$ years while in group B was $46 \pm 17$ years with $\mathrm{p}=0.561$, which was not statistically significant. ${ }^{1}$ Kharadi et al found that mean age in group A was $54 \pm 15.75$ years while in group $B$ was $52 \pm 14$ years with $p=0.765$, and was not statistically significant. ${ }^{11}$ Jenaw et al study mean age group in group A was $46.55+16$ years while in group B was $45.75+15.73$ years with $p$ value $=0.822$, which was not statistically significant. ${ }^{12}$ Meena et al study mean age group in group $\bar{A}$ was 45.4 years while in group B was 46.5 years, which are comparable. Lionetti et al study mean age group in group $A$ was 51 years while in group B was 53.5 years, which are comparable. ${ }^{6}$ Kim-Fuchs et al study mean age group in group A was 49.23 year while in group B was 50.3 years, which are comparable. ${ }^{13}$ Pierides et al study mean age group in group $A$ was 53.2 years while in group B was 51.2 years, which are comparable. ${ }^{14}$ In our study mean age is between $49.13 \pm 17.29$ years in group $A$ and $48.31 \pm 16.44$ years in group $B$ with $p$ value $=0.83$, which is statistically not significant and it is similar in comparison with other studies. Jeroukhimov et al found that the mean post-operative pain score in Group A was $0.6 \pm 0.25$ and in Group B it was $0.06 \pm 0.15$ with p value is 0.071 , which was statistically not significant. Jenaw et al study mean post-operative pain score in group $\bar{A}$ was $0.30 \pm 0.65$ while in group $B$ was $0.08 \pm 0.27$ with $p$ value $=0.045$, which was statistically significant. ${ }^{12}$ Kharadi et al study mean postoperative pain score in Group A was $0.4 \pm 0.55$ and in Group B it was $0.69 \pm 0.34$ with $p=0.40$, which was statistically not significant. ${ }^{11}$ Meena et al study mean post-operative pain score in group A was $1.23 \pm 1.3$ while in group B was $0.92 \pm 0.9$ with $p$ value $=0.013$, which was statistically significant ${ }^{4}$ In our study, mean post-operative pain score is $1.3 \pm 0.9$ in group $A$ and $0.95 \pm 0.8$ in group $B$ with $p$ value 0.048 , which is statistically significant.

We observed that, lower pain score is reported among patients in group B in comparison to group A. Mesh fixation by absorbable suture material causes less irritation of nerves as compared to mesh fixed by non-absorbable suture material. 
This may contribute to significantly less post-operative pain in the Group B compared to Group A. In the study of Igor et al found incidence of seroma formation in group $A(n=92)$ was 5 and in group $B(n=92)$ was $3 p=0.561$ which was not statistically significant. ${ }^{1}$ Kharadi et al found incidence of seroma formation in group $A(n=50)$ was 4 and in group $B$ ( $n=50$ ) was $5 \mathrm{p}=0.73$ which was not statistically significant. ${ }^{11}$ Jenaw et al found incidence of seroma formation in group $A$ $(n=40)$ was 8 and in group $B(n=40)$ was $2 p=0.043$ which was statistically significant. ${ }^{12}$ In our study, the incidence of seroma formation in group $A(n=76)$ is 6 and in group $B(n=76)$ is 9 with p value of 0.69 , which is statistically insignificant. Kharadi et al observed the incidence of scrotal edema in Group $A(n=50)$ was 7 and in Group B $(n=50) 6$ with p value of 0.77 , which was statistically not significant. ${ }^{11}$ In our study the incidence of scrotal edema is 7 in group $A(n=76)$ and 6 in group $B(n=76)$. The $p$ value is 0.59 , with no statistical significance. The edema is due to the dissection around the sac of hernia and handling of tissues while separating the spermatic cord from sac. On ultrasound, epididymo-orchitis was found in 7 patients out of 76 in group $A$ and 6 out of 76 patients in group B. tab. Chymoral Forte (trypsin and chymotrypsin 1,00,000 AU) was given thrice a day to the patients of both groups along with and scrotal elevation. None of the patient required re-exploration. In the study of Igor et al, incidence of wound infection in group $A$ $(n=92)$ was 1 and in group $B(n=92)$ was 2 with $p$ value of 0.561 , which was statistically not significant. ${ }^{1}$ Kharadi et al found no incidence of wound infection in both the study groups during study. Jenaw et al, incidence of wound infection in group $A(n=40)$ was 6 and in group $B(n=40)$ was $l$ with $p$ value of 0.048 , which was statistically significant. ${ }^{11}$ In our study, 3 cases developed infection in group $A(n=76)$ and 6 in group $B$ $(n=76)$ with $p$ value of 0.67 , which suggests no statistical difference in occurrence of wound infection. Patients with wound infection were managed by drainage of collection by opening one or two sutures, pus culture was sent. Oral antibiotics were given according to the culture. None of the patient required wound re-exploration or removal of the mesh. No incidence of local swelling (recurrence) found in early postoperative periods in all the study groups. Igor et al found the incidence of chronic pain in Group $A(n=92)$ was 29 and in Group B $(n=92)$ it was 26, which was statistically not significant. ${ }^{1}$ Kharadi et al found the incidence of chronic pain in Group $A(n=50)$ was 4 and in Group B $(n=50)$ it was 2, which was statistically not significant. ${ }^{11}$ Jenaw et al found the incidence of chronic pain in Group A $(n=40)$ was 8 and in Group B $(n=40)$ it was 2, which was statistically significant. ${ }^{12}$ Lionetti et al found the incidence of chronic pain in Group $A$ $(n=72)$ was 6 and in Group B $(n=72)$ it was 0 , with $p$ value $<0.001$ which was statistically significant. ${ }^{6,7}$ In our study incidence of chronic pain in group $A(n=76)$ was 10 and 3 in group B ( $n=76$ ) with p value 0.048 . It is found to be statistically significant. In our study, an internationally accepted standard definition of pain (pain beyond 3 months) was used. Igor et al found the incidence of recurrence in Group $A(n=92)$ was 2 and in Group B ( $n=92)$ it was 6, which was statistically not significant. ${ }^{1}$ Kharadi et al found the incidence of recurrence in Group A ( $n=50)$ was 2 and in Group B ( $=50)$ it was 1, which was statistically not significant. ${ }^{11}$ Lionetti et al found recurrence of 2 cases in both groups. ${ }^{6}$ Early recurrence is usually due to operation related factors like, tissue tension while suturing, suture material used, way of dealing with the sac (either invagination or ligation and excision), type of hernia repair, post-operative infection and other postoperative complications (hematoma, seroma) and at the last experience of the surgeon. ${ }^{5}$ Late recurrences are mostly due to patients factors like collagen defects that leads to thinning of scar tissue and continued weakness to inguinal floor, age and medical co-morbidities. size, different surgical teams, follow-up period was around 3 months, which is a short period for evaluation of recurrence and hence the results are only showing early recurrence but late recurrence cannot be analyzed.

\section{CONCLUSION}

We conclude that patients with absorbable suture for mesh fixation has less groin pain as compared to non-absorbable suture in Lichtenstein hernioplasty during 3 months follow up period. However, complications like seroma, wound infection comparable in both the groups and are not statistically significant. There is no incidence of early recurrence in both the study groups. Large, multi-centric and long follow up study are needed to confirm the results of our study.

Funding: No funding sources

Conflict of interest: None declared

Ethical approval: The study was approved by the Institutional Ethics Committee

\section{REFERENCES}

1. Igor J, Wiser I, Karasic E, Nesterenko V, Poluksht N, Lavy R, et al. Reduced pain after tension free inguinal hernia repair using absorbable sutures: a single blind randomized clinical trial. J Am Coll Surg. 2014;218:102-7.

2. Mui WL, Ng CS, Fung TM, Cheung FK, Wong CM, Ma TH. Prophylactic ilioinguinal neurectomy in open inguinal hernia repair: a double-blind randomized controlled trial. Ann Surg. 2006:244:27-33.

3. Aasvang E, Kehlet H. Surgical management of chronic pain after inguinal hernia repair. Br J Surg. 2005;92:795-801.

4. Meena LN, Bansal S, Verma P, Rai R. A comparative study of postoperative chronic pain after tension free inguinal hernia repair using absorbable versus non absorbable sutures for mesh fixation. Int Surg J. 2018;5(4):1378-81.

5. Paajanen H, Kossi J, Silvasti S, Hulmi T, Hakala T. Randomized clinical trial of tissue glue versus absorbable sutures for mesh fixation in local anaesthetic Lichtenstein hernia repair. Br J Surg. 2011;98:1245-51

6. Lionetti R, Neola B, Dilillo S, Bruzzese D, Ferulano GP. Sutureless hernioplasty with light-weight mesh and fibrin glue versus Lichtenstein procedure: a comparison of outcomes focusing on chronic postoperative pain. Hernia. 2012;16:127-31

7. Smedberg SG, Broome AE, Gulmo A. Ligation of the hernia sac? Surg Clin North Am. 1984;64(2):299-306.

8. Wagner J, Brunicardi F, Amid P, Chen D. Inguinal hernias. In: Schwartz S, Brunicardi F, Andersen D, Billiar T, Dunn D, Hunter J, eds. Schwartz's principles of surgery. 10th ed. New York: McGraw-Hill Education; 2015: 14951516 .

9. Abramson JH, Gofin J, Hopp C, Makler A, Epstein LM. The epidemiology of inguinal hernia. A survey in western Jerusalem. J Epidemiol Community Health. 1978:32:59.

10. Abrahamson J. Etiology and pathophysiology of primary and recurrent groin hernia formation. Surg Clin North Am. 1998;78(6):953-72.

11. Kharadi A, Shah V. Comparative study of mesh fixation with non-absorbable $\mathrm{v} / \mathrm{s}$ delayed absorbable suture in open inguinal hernia. Int Surg J. 2016;3(3):1180-3.

12. Shanoo A, Sanjeev C, Zeeshan A, Ashish M, Amit G, Jeevan K, et al. Comparative study between monofilament absorbable vs non-absorbable sutures for mesh fixation in Lichtenstein's hernia repair. The Antiseptic. 2017;114:27-31.

13. Kim-Fuchs C, Angst E, Vorburger S, Helbling C, Candinas D, Schlumpf R. Prospective randomized trial comparing sutured with sutureless mesh fixation for Lichtenstein hernia repair: long-term results. Hernia. 2012;16:21-7.

14. Pierides G, Scheinin T, Remes V, Hermunen K, Vironen J. Randomized comparison of self-fixating and sutured mesh in open inguinal hernia repair. Br J Surg. 2012;99:630-6.

\section{LIMITATIONS}

We found certain limitations in our study like small sample 